\title{
Erythrocyte membrane docosapentaenoic acid levels are associated with islet autoimmunity: the Diabetes Autoimmunity Study in the Young
}

\author{
Jill M. Norris • Miranda Kroehl • Tasha E. Fingerlin • \\ Brittni N. Frederiksen • Jennifer Seifert • Randall Wong • \\ Michael Clare-Salzler • Marian Rewers
}

Received: 7 August 2013 / Accepted: 22 October 2013 /Published online: 16 November 2013

(C) Springer-Verlag Berlin Heidelberg 2013

\begin{abstract}
Aims/hypotheses We previously reported that lower $n-3$ fatty acid intake and levels in erythrocyte membranes were associated with increased risk of islet autoimmunity (IA) but not progression to type 1 diabetes in children at increased risk for diabetes. We hypothesise that specific $n$ - 3 fatty acids and genetic markers contribute synergistically to this increased risk of IA in the Diabetes Autoimmunity Study in the Young (DAISY).

Methods DAISY is following 2,547 children at increased risk for type 1 diabetes for the development of IA, defined as being positive for glutamic acid decarboxylase $(\mathrm{GAD})_{65}$, IA-2 or insulin autoantibodies on two consecutive visits. Using a casecohort design, erythrocyte membrane fatty acids and dietary intake were measured prospectively in 58 IA-positive children and 299 IA-negative children.
\end{abstract}

Electronic supplementary material The online version of this article (doi:10.1007/s00125-013-3106-7) contains peer-reviewed but unedited supplementary material, which is available to authorised users.

J. M. Norris $(\bowtie) \cdot$ T. E. Fingerlin $\cdot$ B. N. Frederiksen $\cdot$ J. Seifert Department of Epidemiology, Colorado School of Public Health, University of Colorado Anschutz Medical Campus, 13001 E. 17th Place, Campus Box B119, Aurora, CO 80045, USA

e-mail: Jill.Norris@ucdenver.edu

M. Kroehl • T. E. Fingerlin

Department of Biostatistics and Informatics, Colorado School of Public Health, University of Colorado Anschutz Medical Campus, Aurora, CO, USA

R. Wong $\cdot$ M. Rewers

Barbara Davis Center for Childhood Diabetes, University of

Colorado Anschutz Medical Campus, Aurora, CO, USA

M. Clare-Salzler

Department of Pathology, Immunology and Laboratory Medicine,

University of Florida College of Medicine, Gainesville, FL, USA
Results Lower membrane levels of the $n$ - 3 fatty acid, docosapentaenoic acid (DPA), were predictive of IA (HR 0.23 ; 95\% CI 0.09, 0.55), while $\alpha$-linolenic acid (ALA), eicosapentaenoic acid (EPA) and docosahexaenoic acid (DHA) were not, adjusting for HLA and diabetes family history. We examined whether the effect of dietary intake of the $n-3$ fatty acid ALA on IA risk was modified by fatty acid elongation and desaturation genes. Adjusting for HLA, diabetes family history, ethnicity, energy intake and questionnaire type, ALA intake was significantly more protective for IA in the presence of an increasing number of minor alleles at FADS1 rs174556 ( interaction $=0.017)$, at FADS2 $\mathrm{rs} 174570$ ( $_{\text {interaction }}=$ 0.016 ) and at $F A D S 2$ rs 174583 ( interaction $=0.045$ ).

Conclusions/interpretation The putative protective effect of $n$-3 fatty acids on IA may result from a complex interaction between intake and genetically controlled fatty acid desaturation.

Keywords Autoimmunity · Biomarker · Diet .

Docosapentaenoic acid · Epidemiology $\cdot$ Gene-environment interaction $\cdot$ Genetics $\cdot n-3$ Fatty acids $\cdot n-6$ Fatty acids $\cdot$ Type 1 diabetes

$\begin{array}{ll}\text { Abbreviations } \\ \text { ALA } & \alpha \text {-Linolenic acid } \\ \text { ARA } & \text { Arachidonic acid } \\ \text { COX } & \text { Cyclooxygenase } \\ \text { DAISY } & \text { Diabetes Autoimmunity Study in the Young } \\ \text { DHA } & \text { Docosahexaenoic acid } \\ \text { DPA } & \text { Docosapentaenoic acid } \\ \text { ELOVL2 } & \text { Elongation of very long (chain fatty acids) } 2 \\ \text { ELOVL5 } & \text { Elongation of very long (chain fatty acids) } 5 \\ \text { EPA } & \text { Eicosapentaenoic acid } \\ \text { FADS1 } & \text { Fatty acid desaturase 1 } \\ \text { FADS2 } & \text { Fatty acid desaturase 2 }\end{array}$




$\begin{array}{ll}\text { FFQ } & \text { Food frequency questionnaire } \\ \text { GAD }_{65} & \text { Glutamic acid decarboxylase-65 } \\ \text { GLA } & \gamma \text {-Linolenic acid } \\ \text { IA } & \text { Islet autoimmunity } \\ \text { IA-2 } & \text { Insulinoma antigen 2 } \\ \text { LA } & \text { Linoleic acid } \\ \text { LOX } & \text { Lipooxygenase } \\ \text { PUFA } & \text { Polyunsaturated fatty acid } \\ \text { SNP } & \text { Single nucleotide polymorphism } \\ \text { YAQ } & \text { Youth/Adolescent Questionnaire }\end{array}$

\section{Introduction}

Type 1 diabetes is an autoimmune disease characterised by the destruction of the islet cells of the pancreas. Islet autoimmunity (IA) precedes and is strongly predictive of type 1 diabetes development, and it is likely that both genetic and environmental factors play a role in the development of IA and subsequent progression to type 1 diabetes.

Factors that either promote or inhibit IA may be pivotal in the development of type 1 diabetes. A multitude of studies have shown the substantial effect of $n$ - 3 fatty acids on inflammatory responses in both human and animal populations [1-4] and, as such, a relative deficiency of $n-3$ fatty acids, common to Western diets, may lead to heightened inflammatory reactions and thus increase the risk for autoimmune diseases.

$\alpha$-Linolenic acid (ALA) is the principal $n$ - 3 fatty acid in Western diets, and is found in the green leaves of plants, and in flax, canola, walnuts and soy. The next most common $n-3$ fatty acids are eicosapentaenoic acid (EPA) and docosahexaenoic acid (DHA), which are found in fatty fish. Docosapentaenoic acid (DPA) is less common in Western diets, as its primary source is seal oil, although it is found in small quantities in fish. ALA may serve in a limited capacity as a precursor for EPA and DPA through the work of the desaturation and elongation enzymes, $\delta$-6-desaturase, $\delta-5$ desaturase and elongase, as shown in Fig. 1. Linoleic acid (LA) is the most abundant $n$ - 6 fatty acid in the diet, and is found primarily in nut, seed and vegetable oils. $\gamma$-Linolenic acid (GLA) is uncommon in the diet, with its primary sources being evening primrose oil, borage oil and blackcurrant seed oil. The $n-6$ fatty acid, arachidonic acid, is found in meat and poultry and, like GLA, can be derived from LA via desaturation and elongation.

In the Diabetes Autoimmunity Study in the Young (DAISY), a longitudinal observational study following children at increased risk of developing type 1 diabetes, we reported that both higher dietary intake of total $n$ - 3 fatty acids and a higher percentage of $n-3$ fatty acids in the erythrocyte membrane were associated with a lower risk of IA [5]. We noted that these associations were not seen when we limited

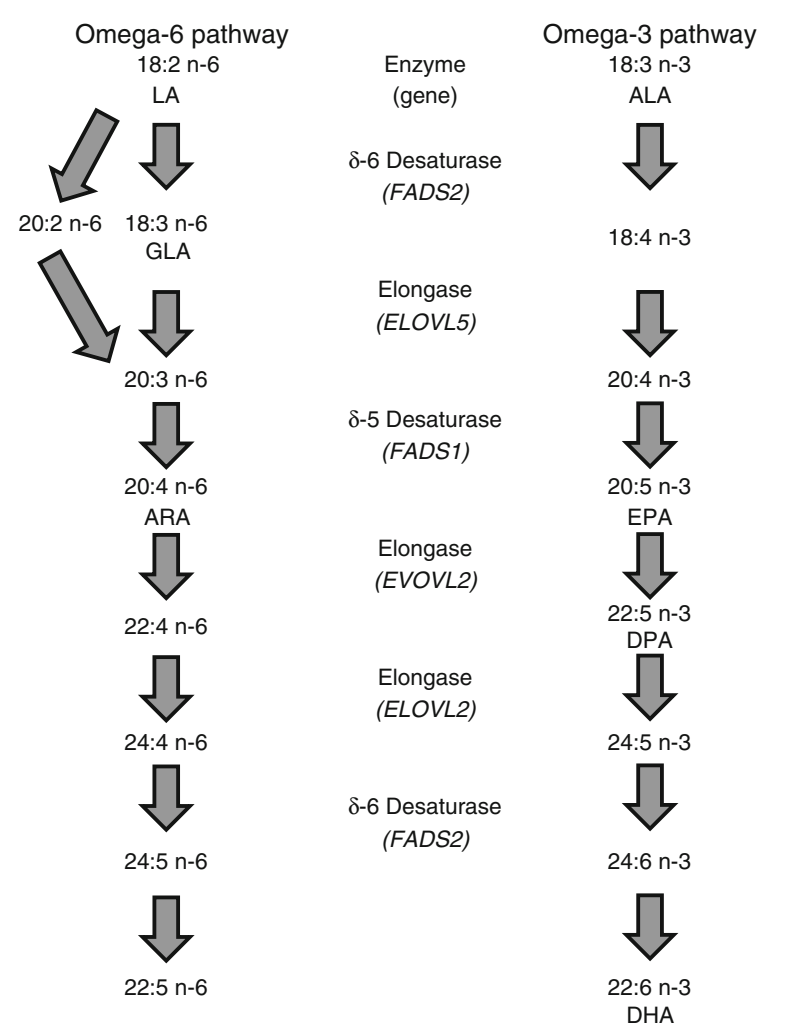

Fig. 1 Scheme of the metabolic pathway of n-6 and n-3 long-chain polyunsaturated fatty acids

the exposure to the putatively more anti-inflammatory $n$-3 fatty acids, EPA and DHA, which was intriguing given that previous studies showed that children with type 1 diabetes were less likely to have received cod liver oil in infancy [6] and had a decreased percentage of DHA in erythrocyte membranes [7] compared with controls.

We further explored individual fatty acids as well as possible gene-environment interactions to elucidate the potential mechanism of our original association with IA [5], with a larger study population and the addition of genetic information on elongation and desaturation enzyme genes. As our previous studies showed that polyunsaturated fatty acid intake and erythrocyte membrane levels were not associated with progression to type 1 diabetes in children with IA [8], we limited these analyses to the outcome of development of IA.

\section{Methods}

\section{DAISY population}

Children are enrolled in DAISY in one of two ways. First, umbilical cord blood was collected and screened for diabetessusceptibility alleles in the HLA region from babies born at $\mathrm{St}$ Joseph's Hospital in Denver, Colorado, a population that is representative of the general population of the Denver 
metropolitan area. Children who were at elevated risk of type 1 diabetes based on the screening results were offered enrolment in the study. Second, children from birth to 8 years of age from the Denver area with first degree relatives with type 1 diabetes, were also eligible for enrolment in the study. PCRbased HLA class II typing on cord blood or the first available blood sample was performed by Roche Molecular Systems, Alameda, CA, USA. Details on the newborn screening [9] and follow-up [5] have been published elsewhere. Participant recruitment took place between 1993 and 2006. Written informed consent was obtained from the parents of study children; assent was obtained from children age 7 years and older. All study protocols were approved by the Colorado Multiple Institutional Review Board.

\section{Measurement of autoantibodies}

Autoantibodies were tested at 9, 15 and 24 months, and annually thereafter, or at the first visit and then annually, depending on enrolment group. Radioimmunoassays were used to measure serum autoantibodies to insulin, glutamic acid decarboxylase (GAD) 65 , and insulinoma antigen 2 (IA-2) (also known as BDC512), as previously described [10-12], with rigorous duplicate testing and confirmation of all positive and a subset of negative results. The cut-off for positivity was established as the 99th percentile of healthy controls. If a subject tested autoantibody positive they were put on an accelerated testing schedule of every 3-6 months. IA is defined as testing positive for at least one of the three autoantibodies on at least two consecutive visits less than 6 months apart (IA case).

Study 1. Erythrocyte membrane PUFA levels and risk of IA

The DAISY cohort contains 2,547 children (Fig. 2). A casecohort design was used to investigate whether membrane levels of polyunsaturated fatty acids (PUFA) were associated with risk for the development of IA. To conduct the DAISY case-cohort study, a representative sample of 380 children (i.e. subcohort) was selected from the DAISY cohort via stratified random sampling based on HLA-DR genotype and family history of type 1 diabetes. During follow-up (1997-2011), 24 children developed IA within the subcohort. We supplemented this with 85 children who developed IA outside this subcohort, to make the full DAISY case-cohort population. We do not have erythrocyte data on all children in the casecohort population because we started collecting erythrocytes at each DAISY visit in 2001 and obtained membrane PUFA composition analyses on these samples only up to 2008 . Therefore, the analysis population of Study 1 includes only those children for whom we obtained erythrocyte membrane PUFA data; 313 children in the subcohort, 14 children who developed IA within the subcohort, and 44 children who developed IA outside this subcohort (Fig. 2). The median number of clinical visits per child at which autoantibody status was ascertained was seven. The median time that elapsed between clinical visits was 367 days.

In order to explore whether PUFA were associated with appearance of a specific autoantibody, we further defined our IA cases according to which autoantibody was positive on the first positive visit. There were 20 children positive for only insulin autoantibodies (IAA), 22 positive for only $\mathrm{GAD}_{65}$ autoantibodies (GAA), and three positive for only IA-2 on their first IA-positive visit. Six were positive for GAA and IAA, one was positive for GAA and IA-2, two were positive for IAA and IA-2, and four were positive for GAA, IAA and IA-2 on their first IA-positive visit. Therefore, there were a total of 33 IA cases that were positive for GAA initially (IA-GAA), 32 IA cases who were positive for IAA initially (IA-IAA), and ten IA cases who were positive for IA-2 initially (IA-IA2).

Measurement of membrane fatty acids Erythrocytes from the blood sample were separated within 30 min of blood draw, flash frozen in liquid nitrogen and stored at $-70^{\circ} \mathrm{C}$. Lipids were extracted from samples of erythrocytes following the method developed by Bligh and Dyer [13], and stored at $-20^{\circ} \mathrm{C}$ in sealed cryotubes following flushing with nitrogen gas. The fatty acids present in the lipid isolates were subsequently methylated using the base-catalysed procedures designed by Maxwell and Marmer [14] in preparation for analysis by gas chromatography (Hewlett-Packard 6890; Agilent, Santa Clara, CA, USA) with mass spectral detection (HewlettPackard 5973; Agilent). The samples, separated across a CPWax column $(25 \mathrm{~m} \times 0.25 \mathrm{~mm}$ internal diameter, $0.2 \mu \mathrm{m}$ film; Varian, Palo Alto, CA, USA), were identified by comparing the retention times and $\mathrm{m} / \mathrm{z}$ of selected ions from analytes in the samples with those of authentic standards (NuCheckPrep; Elysian, MN, USA; Supelco; St Louis, MO, USA). Quantification was determined against five-point standard curves and fatty acid percentage is reported as a per cent of total lipids ( $\mathrm{g}$ of fatty acid/100 g erythrocyte lipid).

We analysed the following fatty acids in the membranes: LA (18:2n-6), GLA (18:3n-6), arachidonic acid (ARA) (20:4n-6), ALA (18:3n-3), EPA (20:5n-3), DHA (22:6n-3), and DPA (22:5n-3). ALA, EPA, DPA and DHA were combined to estimate total $n-3$ fatty acids in the membranes (as a per cent of total lipids), and LA, GLA and ARA were combined to estimate total membrane $n-6$ fatty acids.

Study 2. Dietary $n-3 \times$ gene interaction and risk of IA

In order to examine whether dietary intake of $n$ - 3 fatty acids interacts with fatty acid desaturase and elongation genes to affect risk of IA, we used the same DAISY case-cohort as 
Fig. 2 Description of analysis populations used in Study 1 and Study 2 from the DAISY

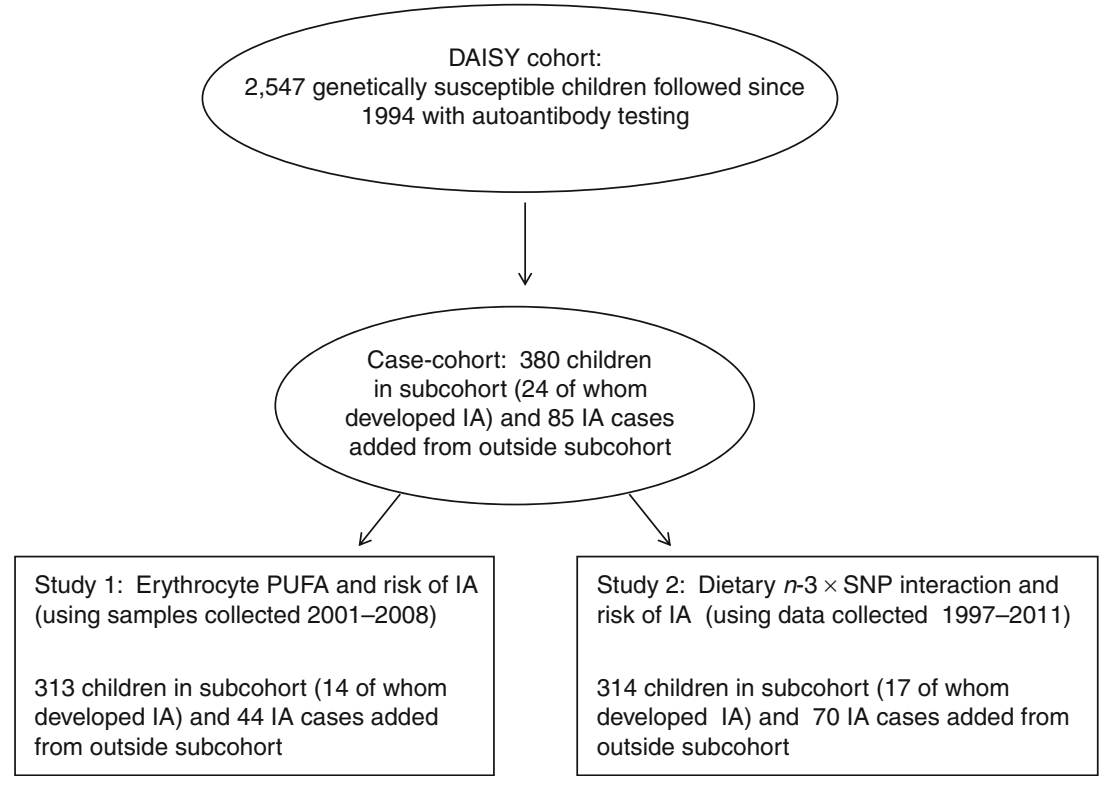

described earlier. Of the 465 children in the case-cohort, complete genotype and dietary data (as described below) were collected between 1997 and 2011 and were available for 384 children (87 IA-positive and 297 IA-negative); these were included in Study 2 (Fig. 2). While the analysis populations used in both Study 1 and Study 2 were derived from the same case-cohort in DAISY, differences in participant numbers can be explained by sample and dietary data availability. Electronic supplementary material (ESM) Fig. 1 shows the overlap between the two study populations. Table 1 shows that the characteristics of the children included in Study 1 and Study 2 are similar, despite differences in calendar year of data collection.

\section{Collection and analysis of supplement and dietary} intake Parents of children in the DAISY cohort complete an annual interview in which data on supplements taken in the last year, including those containing $n-3$ fatty acids, is collected. To complement the annual interview, we prospectively measured early childhood diet using a 111-item semiquantitative food frequency questionnaire (FFQ). This questionnaire has been specifically designed for use in pre-school children, and validated against multiple $24 \mathrm{~h}$ recalls in the DAISY population [15]. The FFQs are administered annually beginning at the age of 2 years, or at enrolment if after the age of 2 years. Parents are asked to recall the diets of their children in the previous year for children aged 2-10 years. Starting between the ages of 10 and 12 years, children are asked to recall their own diets and complete the Youth/Adolescent Questionnaire (YAQ), an FFQ geared toward adolescents $[16,17]$. This questionnaire was developed from the aforementioned FFQ and, in a comparison study in our DAISY population, we determined that data from these two instruments may be combined when an instrument indicator variable (i.e. whether data were collected by FFQ or YAQ ['FFQ type']), was included in the model [18]. In our analysis, antibody and dietary data were linked if the data that were collected represented the dietary intake of the time directly preceding the clinic visit at which the autoantibody data was measured. A quantitative dietary assessment of PUFA intake was not available for the first year of life in DAISY; therefore we do not have intake data during infancy. This was an observational study; no dietary advice was given to the families.

$n-3$ and $n-6$ fatty acids and other nutrient intakes were measured in both the FFQ and YAQ based on a commonly used unit or portion size for each food (e.g., one egg or 84-112 g (3-4 oz.) of fish, etc.), which was specified on the FFQ and the parents were asked how often on average during the previous year their child had consumed that amount. Nine responses were possible, ranging from 'never' to ' $\geq 6$ times /day'. Specifically, the questionnaire asked about the frequency of intake of canned tuna, dark-meat fish (mackerel, salmon, sardines, bluefish and swordfish), other fish (not specified), and shrimp, lobster and scallops. The questionnaire also inquired about the kind of fat usually used for frying, sautéing, and baking (vegetable oil, solid vegetable oil shortening, butter, margarine, lard or none). The intake of nutrients was computed for each child by multiplying the frequency of consumption of each unit of food by the nutrient content of the specified portions. Composition values for fatty acids and other nutrients were obtained from the Harvard University Food Composition Database, as derived from US Department of Agriculture sources [19] and supplemented by manufacturer information [20]. The calculation of EPA and DHA intake from this FFQ and database is described in detail 
Table 1 Descriptive characteristics of children in Study 1 and Study 2

\begin{tabular}{|c|c|c|c|c|}
\hline Variable & IA cases ${ }^{\mathrm{a}}$ & IA-negative children & Unadjusted HR (95\% CI) & $p$ value \\
\hline \multicolumn{5}{|l|}{ Study 1: Erythrocyte PUFA and risk of IA } \\
\hline$n$ & 58 & 299 & $\mathrm{~N} / \mathrm{A}$ & \\
\hline Mean age ${ }^{\mathrm{b}}$, years; mean (SD) & $5.4(3.3)$ & $8.2(3.6)$ & N/A & \\
\hline Median year of $1^{\text {st }}$ IA positivity (range) & 2005 (2001-2008) & $\mathrm{N} / \mathrm{A}$ & N/A & \\
\hline HLA-DRB1*03/DRB1*04,DQB1*0302 & $20(34.5)$ & $83(27.8)$ & $1.26(0.66,2.41)$ & 0.49 \\
\hline Family history of type 1 diabetes & $30(51.7)$ & $117(39.1)$ & $1.45(0.73,2.88)$ & 0.28 \\
\hline Female & $34(58.6)$ & $148(49.5)$ & $1.51(0.84,2.71)$ & 0.17 \\
\hline Non-Hispanic white ethnicity & $46(79.3)$ & $222(74.3)$ & $1.15(0.56,2.35)$ & 0.71 \\
\hline Maternal education $>12$ years ${ }^{\mathrm{c}}$ & $51(87.9)$ & $234(78.8)$ & $1.69(0.65,4.40)$ & 0.28 \\
\hline Maternal age, years; mean $(\mathrm{SD})^{\mathrm{d}}$ & $30.5(5.5)$ & $30.0(5.4)$ & $1.00(0.95,1.06)$ & 0.91 \\
\hline \multicolumn{5}{|c|}{ Study 2: Dietary $n-3 \times$ SNP interaction and risk of IA } \\
\hline$n$ & 87 & 297 & $\mathrm{~N} / \mathrm{A}$ & \\
\hline Mean age ${ }^{\mathrm{b}}$, years; mean $(\mathrm{SD})$ & $6.0(3.1)$ & $9.6(4.3)$ & $\mathrm{N} / \mathrm{A}$ & \\
\hline Median year of $1^{\text {st }}$ IA positivity (range) & $2005(1997-2011)$ & $\mathrm{N} / \mathrm{A}$ & $\mathrm{N} / \mathrm{A}$ & \\
\hline HLA-DRB1*03/DRB1*04,DQB1*0302 & $32(36.8)$ & $78(26.3)$ & $1.49(0.86,2.57)$ & 0.15 \\
\hline Family history of type 1 diabetes & $49(56.3)$ & $102(34.3)$ & $2.49(1.48,4.19)$ & 0.001 \\
\hline Female & $43(49.4)$ & $144(48.5)$ & $1.11(0.66,1.85)$ & 0.70 \\
\hline Non-Hispanic white ethnicity & $69(79.3)$ & $219(73.7)$ & $1.03(0.55,1.94)$ & 0.92 \\
\hline Maternal education $>12$ years $^{\mathrm{c}}$ & $76(87.4)$ & $230(77.7)$ & $1.62(0.74,3.52)$ & 0.23 \\
\hline Maternal age, years; mean $(\mathrm{SD})^{\mathrm{d}}$ & $30.9(5.2)$ & $29.7(5.4)$ & $1.03(0.98,1.08)$ & 0.24 \\
\hline
\end{tabular}

Data are $n(\%)$ except where otherwise stated

${ }^{a}$ In Study 1, 14 cases developed from within the subcohort and 44 cases were added from outside the subcohort. In Study 2, 17 cases developed from within the subcohort and 70 cases were added from outside the subcohort

${ }^{\mathrm{b}}$ Age at first IA-positive visit or at last follow-up if IA negative

${ }^{\mathrm{c}}$ Data on maternal education were missing in two children

${ }^{\mathrm{d}}$ Data on maternal age were missing in three children

elsewhere [21]. We found that intake of $n-3$ fatty acids as assessed by our FFQ was correlated with erythrocyte membrane composition of the same fatty acids in DAISY children over time, with a stronger correlation for EPA and DHA combined $(r=0.42, p<0.0001)$ than for all $n-3$ fatty acids combined (ALA, EPA, DPA and DHA) $(r=0.23, p<0.0001)$ [22].

Genotyping We genotyped single nucleotide polymorphisms (SNPs) in genes encoding elongation and fatty acid desaturation enzymes [23], which have also been found to be associated with either plasma or membrane PUFAs [24-27]. Four SNPs in the elongation gene, ELOVL2 (rs1570069, rs7744440, rs3798719, rs953413), and four SNPs in the desaturation gene cluster FADS1/ FADS2 (rs174537, rs174556, rs174570, rs174583) were genotyped using the TaqMan SNP genotype based OpenArray platform (Applied Biosystems, Carlsbad, CA, USA). Custom designed 48-sample arrays and normalised genomic DNA were loaded using the OpenArray AccuFill system and cycling was performed on a GeneAmp 9700 PCR system (Applied
Biosystems), all according to manufacturer protocol. Alleles were analysed using the OpenArray SNP genotyping analysis software v.1.0.3 and TaqMan Genotyper Software 2.0 (Applied Biosystems). ESM Table 1 shows the minor allele frequencies of the eight SNPs in the DAISY subcohort.

Statistical analysis

All analyses were conducted in SAS for Windows Version 9.3 (SAS Institute, Cary, NC, USA). Using Cox regression analysis, HRs and $95 \%$ CIs were estimated for the risk of IA for a one SD difference in membrane PUFA. SDs used for this standardisation technique are listed in the footnote of the relevant table and figure. A clustered time-to-event analysis was performed treating siblings from the same family as clusters, and robust sandwich variance estimates [28] were used for statistical inference. Exposure measures prior to onset of IA were available for all children to determine time-toevent. As membrane PUFA and dietary intake were measured longitudinally, we treated them as time-varying in our 
analyses, such that levels/amounts could vary with the clinical visits and reflect change over time in children who were still at risk of IA at a given event time. To account for the sampling of the case-cohort design, the analyses were weighted using the Barlow method [29] and a SAS macro developed by Barlow et al [30]. Models in Study 1 were adjusted for family history of type 1 diabetes and HLA-DRB $1 * 03 / \mathrm{DRB} 1 * 04$, DQB1*0302 genotype. Models in Study 2 were additionally adjusted for caloric intake (kcal/day), type of questionnaire (FFQ vs YAQ) and ethnicity (non-Hispanic white vs other).

Our primary outcome was IA. In Study 1, we also tested a secondary outcome identifying the autoantibody that was present at the first positive visit, IA-IAA, IA-GAA and IAIA2. This did not alter the IA event time, but only counted the event if the specified autoantibody was present at the first positive visit; in some cases there was more than one autoantibody present at the first positive visit.

The SNPs in the elongation and desaturation genes were analysed additively. For the a priori interaction models, we created an interaction term between each of the selected SNPs and dietary n-3 fatty acid intake. Each interaction term was tested in separate adjusted models. The four SNPs in the FADS1/FADS2 cluster and the four SNPs in the ELOVL2 gene were in linkage disequilibrium $(0.3<r<0.96)$ (see ESM Fig. 2). Statistical significance was defined by a twosided alpha level of $5 \%$. As we based our analyses on a priori hypotheses, previous observations and targeted data collection, we did not correct for multiple comparisons, as recommended by Rothman [31].

\section{Results}

Study 1. Erythrocyte membrane PUFA levels and risk of IA

In the Study 1 population, the mean age of IA development was 5.4 years and the mean age at last follow-up in children who were IA negative was 8.2 years (Table 1). In order to describe the cohort's erythrocyte membrane fatty acid composition, which was collected at multiple time points throughout childhood, we present mean $( \pm \mathrm{SD})$ levels at three ages (3, 6 and 9 years) for children in Study 1 (Table 2).

Higher levels of DPA in the erythrocyte membrane, as a per cent of total lipids, were associated with a decreased risk of IA, adjusting for HLA-DRB1 status and family history of type 1 diabetes (Table 3 ). Total membrane $n$ - 3 fatty acids were also associated with decreased risk of IA, although this appears to be largely driven by DPA, as the other $n$ - 3 fatty acids, ALA, EPA and DHA, were not associated with IA. None of the membrane $n-6$ fatty acids was associated with risk of IA.

We then examined whether DPA levels were associated with IA seroconversion characterised by the autoantibody that was present first: insulin (IA-IAA), GAD $_{65}$ (IA-GAA) and
Table 2 Description of $n-3$ and $n-6$ erythrocyte fatty acid content at three ages in children in the case-cohort

\begin{tabular}{llll}
\hline & \multicolumn{2}{l}{ Group of children ${ }^{\mathrm{a}}$, mean $(\mathrm{SD})$} \\
\cline { 2 - 4 } Erythrocyte fatty acid levels & 3-year-olds & 6-year-olds & 9-year-olds \\
\hline$n$ & 111 & 164 & 136 \\
Total $n$-3 fatty acids $^{\mathrm{b}}$ & $4.23(1.34)$ & $4.28(1.40)$ & $4.69(1.67)$ \\
ALA & $0.25(0.12)$ & $0.24(0.12)$ & $0.24(0.22)$ \\
EPA & $0.18(0.08)$ & $0.20(0.12)$ & $0.27(0.20)$ \\
DPA & $1.65(1.05)$ & $1.59(1.00)$ & $1.72(1.22)$ \\
DHA & $2.15(0.61)$ & $2.26(0.76)$ & $2.46(0.80)$ \\
Total $n-6$ fatty acids & $25.35(3.82)$ & $25.47(4.36)$ & $26.42(2.61)$ \\
LA & $13.81(2.65)$ & $13.83(3.03)$ & $14.27(2.06)$ \\
GLA & $0.12(0.06)$ & $0.11(0.06)$ & $0.15(0.06)$ \\
ARA & $11.43(2.12)$ & $11.53(2.04)$ & $11.99(1.49)$ \\
\hline
\end{tabular}

${ }^{\mathrm{a}}$ These data are from cross-sectional analysis of the cohort. The same child may be in one or all of these age group samples depending on whether fatty acid data were available at these ages. Fatty acid content is expressed as percentage of total fatty acid levels. Age groups are determined as follows: 3 -year-olds are at least 3 years of age but less than 4 years of age; 6 -year-olds are at least 6 years of age, but less than 7 years of age; 9 -year-olds are at least 9 years of age, but less than 10 years of age

${ }^{\mathrm{b}}$ Total $n$ - 3 fatty acids variable is a sum of ALA, EPA, DPA and DHA

${ }^{\mathrm{c}}$ Total $n-6$ fatty acids variable is a sum of LA, GLA and ARA

IA-2 (IA-IA2). Membrane DPA level was marginally associated with time to IA-IAA positivity (HR $0.32 ; 95 \%$ CI 0.10 , $1.03 ; p=0.056$ ), and significantly associated with IA-GAA (HR $0.14 ; 95 \%$ CI $0.05,0.39 ; p<0.001$ ) and IA-IA2 (HR 0.06; 95\% CI 0.01, 0.36; $p=0.003$ ).

\section{Study 2. Dietary $n-3 \times$ gene interaction and risk of IA}

The presence of DPA in the erythrocyte membrane is unlikely to be the result of dietary intake of DPA, as it is uncommon in the Western diet, and found primarily in seal oil and, to a much smaller extent, in fish oils. Therefore, dietary DPA intake is not a strong candidate environmental factor for IA. However, erythrocyte membrane DPA level (as a per cent of total lipids) is, in part, determined by dietary intake of ALA and endogenous conversion from this precursor fatty acid by consecutive desaturation and chain elongation (Fig. 1). Therefore, we hypothesised that ALA intake would interact with fatty acid desaturation and elongation gene variants to affect risk of IA.

The description of the Study 2 population is presented in Table 1. We examined four SNPs in the FADS1/FADS2 gene cluster and four SNPs in ELOVL2. The allele frequencies of the SNPs are shown in ESM Table 1. None of the SNPs were associated with IA. In order to describe the dietary $n$-3 fatty acid intake in DAISY, which was collected at multiple time points throughout childhood, Table 4 presents mean $( \pm \mathrm{SD})$ intake (in $\mathrm{g} /$ day) at three ages (3, 6 and 9 years) for children in 
Table 3 Association between erythrocyte membrane $n-3$ or $n-6$ fatty acid content and risk of developing islet autoimmunity in 357 children with erythrocyte fatty acid measures, of whom 58 progressed to IA

\begin{tabular}{llll}
\hline Erythrocyte membrane fatty acid levels & Unadjusted $\mathrm{HR}^{\mathrm{a}}(95 \% \mathrm{CI})$ & $p$ value & Adjusted HR ${ }^{\mathrm{a}, \mathrm{b}}(95 \% \mathrm{CI})$ \\
\hline Total $n-3$ fatty acids $^{\mathrm{c}}$ & $0.60(0.43,0.84)$ & 0.003 & $0.52(0.34,0.79)$ \\
ALA & $0.79(0.47,1.32)$ & 0.37 & $0.77(0.44,1.34)$ \\
EPA & $1.06(0.88,1.28)$ & 0.52 & $1.06(0.88,1.28)$ \\
DPA & $0.35(0.19,0.65)$ & 0.001 & $0.23(0.09,0.55)$ \\
DHA & $0.93(0.70,1.25)$ & 0.65 & $0.91(0.67,1.23)$ \\
Total $n$ - 6 fatty acids ${ }^{\mathrm{d}}$ & $0.81(0.61,1.09)$ & 0.16 & $0.80(0.60,1.08)$ \\
LA & $0.90(0.65,1.25)$ & 0.54 & $0.91(0.65,1.25)$ \\
GLA & $0.90(0.69,1.17)$ & 0.42 & $0.89(0.68,1.16)$ \\
ARA & $0.76(0.57,1.02)$ & 0.07 & $0.74(0.54,1.01)$ \\
\hline
\end{tabular}

${ }^{\mathrm{a}} \mathrm{HR}$ and $95 \% \mathrm{CI}$ represent the relative hazard for a one SD difference in the fatty acid variable. SDs used in this analysis are: 1.56 for total $n-3$ fatty acids, 0.13 for ALA, 0.12 for EPA, 1.21 for DPA, 0.78 for DHA, 3.62 for total $n$ - 6 fatty acids, 2.60 for LA, 0.06 for GLA and 1.89 for ARA. Fatty acids are measured as a per cent of total membrane lipids and are analysed as time-varying covariates

${ }^{\mathrm{b}}$ Adjusted for HLA-DR status and family history of type 1 diabetes

${ }^{\mathrm{c}}$ Total $n-3$ fatty acids variable is the sum of ALA, EPA, DPA and DHA

${ }^{\mathrm{d}}$ Total $n-6$ fatty acids variable is the sum of LA, GLA and ARA

the case-cohort. Intake of ALA was inversely associated with IA (unadjusted HR $0.72,95 \%$ CI $0.55,0.98, p=0.03$ ); none of the other dietary $n$ - 3 fatty acids was associated with IA (data not shown). We then examined whether the effect of ALA intake was modified by variation in fatty acid elongation and desaturation genes, by individually testing interactions between ALA intake as a continuous time-varying covariate and eight SNPs using an additive model, adjusting for HLADRB1 status, family history of type 1 diabetes, ethnicity, total energy intake and FFQ type. We detected significant interactions with SNPs in FADS1 (rs174556) $(p=0.017)$, and FADS2 (rs174570 and rs174583) ( $p=0.016$ and $p=0.045$, respectively). As shown in Fig. 3, ALA intake appears to be more protective for IA in the presence of an increasing number of minor alleles at rs 174556 (HR 0.86, 0.49, 0.28 for $0,1,2$ alleles, respectively), for rs 174570 (HR $0.76,0.35,0.17$ for $0,1,2$ alleles, respectively), and for rs174583 (HR 0.88, 0.58, 0.38 for $0,1,2$ alleles, respectively). Similar interactions were seen when our dietary intake variable was the sum of ALA and EPA (data not shown). As $n-6$ fatty acids compete for the same enzymes for desaturation, we included an $n-6$ fatty acid intake variable (i.e., the sum of LA, GLA and ARA) in the aforementioned models and this did not affect the risk estimates or $p$ values of the interaction terms (data not shown).

\section{Discussion}

Our analysis of children at increased risk of type 1 diabetes shows that increased $n-3$ fatty acid status, indicated by higher membrane levels of DPA, is predictive of lower risk of IA. This inverse association holds, regardless of which autoantibody, GAA, IAA or IA-2, is present on the first positive visit. Moreover, $n$ - 3 fatty acid intake, specifically ALA, in children

Table 4 Description of average daily $n$ - 3 fatty acid intake at three ages in children in the case-cohort

\begin{tabular}{llll}
\hline$n$-3 Fatty acid intake variables (average g/day) & \multicolumn{3}{l}{ Group of children ${ }^{\mathrm{a}}$, mean (SD) } \\
\cline { 2 - 4 } & 3-year-olds & 6-year-olds & 9-year-olds \\
\hline$n$ & 236 & 232 & 162 \\
ALA & $1.01(0.50)$ & $0.97(0.43)$ & $1.07(0.52)$ \\
EPA & $0.035(0.045)$ & $0.048(0.057)$ & $0.042(0.062)$ \\
DPA & $0.018(0.016)$ & $0.020(0.017)$ & $0.018(0.015)$ \\
DHA & $0.090(0.079)$ & $0.11(0.10)$ & $0.092(0.090)$ \\
\hline
\end{tabular}

${ }^{\text {a }}$ These data are from cross-sectional analysis of the cohort. The same child may be in one or all of these age group samples depending on whether fatty acid data were available at these ages. Age groups are determined as follows: 3 -year-olds are at least 3 years of age but less than 4 years of age; 6-yearolds are at least 6 years of age, but less than 7 years of age; 9 -year-olds are at least 9 years of age, but less than 10 years of age 


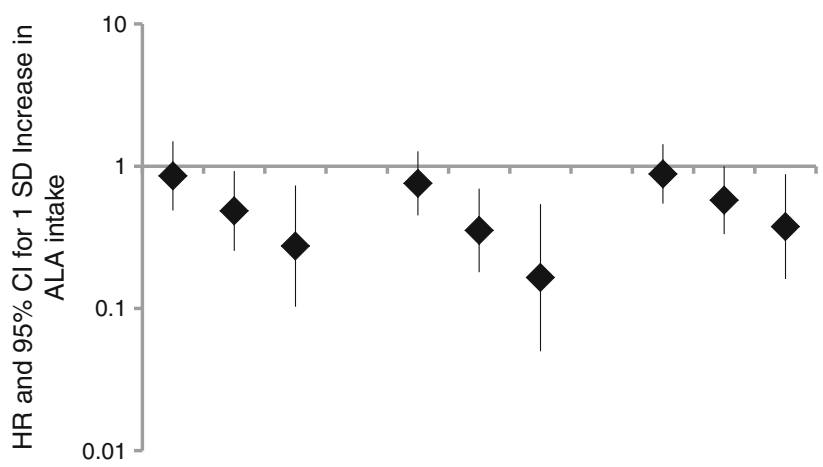

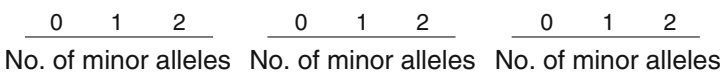
FADS1 rs174556 FADS2 rs174570 FADS2 rs174583

Fig. 3 The decreased risk of IA with increased ALA intake differs by number of FADS1 and FADS2 minor alleles in the case-cohort of DAISY. The analysis cohort consisted of 87 children who developed IA and 297 children who did not. HRs and 95\% CIs represent the relative hazard for a one SD difference in dietary intake. The SD for ALA intake was 0.46. The intake variable was analysed as a time-varying covariate, and the model adjusted for HLA-DRB $1 * 03 / \mathrm{DRB} 1 * 04, \mathrm{DQB} 1 * 0302$, family history of type 1 diabetes, ethnicity, total energy intake and FFQ type. We detected significant interactions between dietary intake and FADS1 (rs174556) $(p=0.017)$, and FADS2 (rs174570 and rs174583) $(p=0.016$ and $p=0.045$, respectively). The HRs and CIs shown in the figure are calculated from the estimates produced by the adjusted models containing the interaction terms

who possess minor alleles in genes encoding fatty acid desaturation enzymes, conveys protection against IA, suggesting that targeted interventions may be possible.

The role of $n-3$ fatty acids in the prevention of IA is not yet understood. Long-chain $n$-3 fatty acids that are incorporated into cell membranes act as substrates for a class of antiinflammatory eicosanoids, resolvins and protectins [32]. These proresolving mediators alter the actions of proinflammatory cytokines, via suppression, acting as competing substrates for arachidonic acid (which produces proinflammatory eicosanoids). The anti-inflammatory effect of these molecules thus reduces oxidative stress. Although less studied than its EPA and DHA counterparts, it is hypothesised that DPA might act as a precursor for production of the DPA-related D-series of resolvins, since it is known to be metabolised by lipooxygenase (LOX) enzymes [33]. Studies suggest that DPA is capable of interfering with the cyclooxygenase (COX) pathway and accelerating the LOX pathway, and as such may be a more potent COX-1 inhibitor than EPA [34]. A recent study reported that, after EPA supplementation, COX inhibition was attributable to DPA activity, suggesting that EPA may exert its anti-inflammatory effects indirectly through elongation to DPA [35].

While DPA has not been previously associated with type 1 diabetes or IA, lower serum/plasma DPA levels have been associated with other health outcomes such as myocardial infarction, cardiovascular events, type 2 diabetes and high blood pressure [36-39]. In our previous report of $n-3$ fatty acids and IA [5], our a priori aim was to compare the effect of total $n$ - 3 fatty acids to EPA and DHA; therefore, at that time, we did not analyse the association between IA and erythrocyte membrane DPA levels alone. Virtanen et al found no association between any of the serum $n-3$ fatty acids, including DPA, and risk of IA [40]. However, this may be due to differences between our measures of fatty acid status. Our study describes the fatty acid composition of erythrocyte membranes, which reflects the intake and metabolism of fatty acids over a period of 4-6 weeks [41], whereas serum levels reflect intake over a period of 1-2 weeks [42-44]. Given that fatty fish, a common source of $n$ - 3 fatty acids, is infrequently consumed by young children, a long-term biomarker of $n-3$ fatty acid intake, as well as an indicator of what is being incorporated into the membranes, may be more suitable for an estimate of fatty acid status in children than a short-term biomarker of intake.

As DPA is uncommon in the diet, its presence in cell membranes is the result of a cascade of dietary intake and enzymatic action (see Fig. 1). $\delta$-6-Desaturase, encoded by FADS2 [23], elongase, encoded by ELOVL5, and $\delta-5$ desaturase, encoded by FADS1 [23], work in series to convert ALA to EPA. EPA is then converted to DPA via an elongase encoded by ELOVL2. There is also the possibility of retroconversion from DPA to EPA [45]. Dietary supplementation with ALA-rich canola, linseed or flaxseed oils resulted in significant increases in platelet phospholipid DPA concentrations, as reviewed in Burdge and Calder [46]. The conversion from dietary ALA to EPA and then to DPA that takes place in the body and that may be partly under genetic control, may explain why IA risk was associated with dietary intake of ALA (univariately and, more strongly, in interaction with FADS variants) and not erythrocyte membrane ALA levels. Notable strengths of this study are the use of two exposure assessment methods, the biomarker of erythrocyte membrane fatty acid content and the measurement of intake via FFQ, in addition to a measure of potential host (genetic) modifiers. As this was an observational study of fatty acid status during usual childhood dietary intake, we cannot infer what associations we would observe with targeted supplementation with DHA or combinations of $n-3$ fatty acids.

The observation that the association between dietary intake of ALA and risk of IA is modified by genetic variation in FADS1 and FADS2 has not been reported previously in type 1 diabetes. However, several studies have shown that genetic variation in FADS1 modifies the association between dietary intake of $n-3$ fatty acids, and ALA alone, on total and nonHDL cholesterol levels in adults and adolescents [47-49], but not children [50].

The ability to differentiate between risk factors for the appearance of IA and the subsequent progression to type 1 diabetes is enabled by successful long-term follow-up of a cohort of children at increased risk for type 1 diabetes, which 
is a major strength of DAISY. The current study, along with the two previous studies in this cohort $[5,8]$ show that $n-3$ fatty acids are more influential on development of IA than progression from IA to type 1 diabetes. The number of IApositive children included in our analysis is limited by the cost of assembling and following such a truly unique cohort. While our study had adequate power to detect the aforementioned novel interactions between dietary intake and variants in $F A D S 1$ and FADS2, it is possible that, due to limited power, interactions of smaller magnitude may exist but were not detected. This is the first study to observe this diet-gene interaction in the development of IA and, in order to dismiss the possibility of type 1 error and lend further evidence to this association, it is important that this be studied in other populations.

Overall, our data suggest that ALA intake throughout childhood in children possessing genetic variants influencing fatty acid desaturation may decrease the risk of IA in those at increased genetic risk for type 1 diabetes. This provides important information regarding the design of studies aimed at preventing the development of type 1 diabetes using PUFA. Our results suggest that analyses of FADS gene variants should be included in cohort and intervention studies addressing the biological effects of long-chain PUFA in order to enhance study sensitivity, precision and inference.

Acknowledgements We thank all the DAISY children and their families who generously volunteered their time and knowledge.

Funding This research was supported by National Institutes of Health grants R01-DK49654, R01-DK32493, and the Diabetes Endocrine Research Center, Clinical Investigation \& Bioinformatics Core P30 DK 57516.

Duality of interest The authors declare that there is no duality of interest associated with this manuscript.

Contribution statement JMN was responsible for the conception of the study, acquisition of data, analysis and interpretation of data and drafting the article. MR and MC-S were responsible for the conception of the study, acquisition of data and revising the manuscript. MK and TEF were responsible for analysis and interpretation of the data and revising the manuscript. BF, JS, and RW were responsible for acquisition of data and revising the manuscript. All authors approved the final version.

\section{References}

1. Calder PC, Grimble RF (2002) Polyunsaturated fatty acids, inflammation and immunity. Eur J Clin Nutr 56(Suppl 3):S14-S19

2. De Caterina R, Madonna R, Massaro M (2004) Effects of omega-3 fatty acids on cytokines and adhesion molecules. Curr Atheroscler Rep 6:485-491

3. Fritsche K (2006) Fatty acids as modulators of the immune response. Annu Rev Nutr 26:45-73

4. Kew S, Mesa MD, Tricon S, Buckley R, Minihane AM, Yaqoob P (2004) Effects of oils rich in eicosapentaenoic and docosahexaenoic acids on immune cell composition and function in healthy humans. Am J Clin Nutr 79:674-681

5. Norris JM, Yin X, Lamb MM et al (2007) Omega-3 polyunsaturated fatty acid intake and islet autoimmunity in children at increased risk for type 1 diabetes. J Am Med Assoc 298:1420-1428

6. Stene LC, Joner G, The Norwegian Childhood Diabetes Study Group (2003) Use of cod liver oil during the first year of life is associated with lower risk of childhood-onset type 1 diabetes: a large, population-based, case-control study. Am J Clin Nutr 78:1128-1134

7. Decsi T, Szabo E, Burus I et al (2007) Low contribution of $n-3$ polyunsaturated fatty acids to plasma and erythrocyte membrane lipids in diabetic young adults. Prostaglandins Leukot Essent Fat Acids 76:159-164

8. Miller MR, Yin X, Seifert J et al (2011) Erythrocyte membrane omega-3 fatty acid levels and omega-3 fatty acid intake are not associated with conversion to type 1 diabetes in children with islet autoimmunity: the Diabetes Autoimmunity Study in the Young (DAISY). Pediatr Diabetes 12:669-675

9. Rewers M, Bugawan TL, Norris JM et al (1996) Newborn screening for HLA markers associated with IDDM: diabetes autoimmunity study in the young (DAISY). Diabetologia 39:807-812

10. Grubin CE, Daniels T, Toivola B et al (1994) A novel radioligand binding assay to determine diagnostic accuracy of isoform-specific glutamic acid decarboxylase antibodies in childhood IDDM. Diabetologia 37:344-350

11. Gianani R, Rabin DU, Verge CF et al (1995) ICA512 autoantibody radioassay. Diabetes 44:1340-1344

12. Yu L, Robles DT, Abiru N et al (2000) Early expression of antiinsulin autoantibodies of humans and the NOD mouse: evidence for early determination of subsequent diabetes. Proc Natl Acad Sci U S A 97:1701-1706

13. Bligh EG, Dyer WJ (1959) A rapid method of total lipid extraction and purification. Can J Biochem Physiol 37:911-917

14. Maxwell RJ, Marmer WN (1983) Systematic protocol for the accumulation of fatty acid data from multiple tissue samples: tissue handling, lipid extraction and class separation, and capillary gas chromatographic analysis. Lipids 18:453-459

15. Parrish LA, Marshall JA, Krebs NF, Rewers M, Norris JM (2003) Validation of a food frequency questionnaire in preschool children. Epidemiology 14:213-217

16. Rockett HR, Wolf AM, Colditz GA (1995) Development and reproducibility of a food frequency questionnaire to assess diets of older children and adolescents. J Am Diet Assoc 95:336-340

17. Rockett HR, Breitenbach M, Frazier AL et al (1997) Validation of a youth/adolescent food frequency questionnaire. Prev Med 26:808816

18. Lamb MM, Ross CA, Brady HL, Norris JM (2007) Comparison of children's diets as reported by the child via the Youth/Adolescent Questionnaire and the parent via the Willett food-frequency questionnaire. Public Health Nutr 10:663-670

19. Haytowitz DB (1995) Information from USDA's Nutrient Data Bank. J Nutr 125:1952-1955

20. Willett WC, Sampson L, Stampfer MJ et al (1985) Reproducibility and validity of a semiquantitative food frequency questionnaire. Am J Epidemiol 122:51-65

21. Iso H, Rexrode KM, Stampfer MJ et al (2001) Intake of fish and omega-3 fatty acids and risk of stroke in women. JAMA 285: 304-312

22. Orton HD, Szabo NJ, Clare-Salzler M, Norris JM (2008) Comparison between omega- 3 and omega- 6 polyunsaturated fatty acid intakes as assessed by a food frequency questionnaire and erythrocyte membrane fatty acid composition in young children. Eur J Clin Nutr 62:733-738

23. Voruganti VS, Higgins PB, Ebbesson SO et al (2012) Variants in CPT1A, FADS1, and FADS2 are associated with higher levels of 
estimated plasma and erythrocyte delta-5 desaturases in Alaskan Eskimos. Front Genet 3:86

24. Tanaka T, Shen J, Abecasis GR et al (2009) Genome-wide association study of plasma polyunsaturated fatty acids in the InCHIANTI Study. PLoS Genet 5:e1000338

25. Rzehak P, Heinrich J, Klopp N et al (2009) Evidence for an association between genetic variants of the fatty acid desaturase 1 fatty acid desaturase 2 ( FADS1 FADS2) gene cluster and the fatty acid composition of erythrocyte membranes. Br J Nutr 101:20-26

26. Malerba G, Schaeffer L, Xumerle L et al (2008) SNPs of the FADS gene cluster are associated with polyunsaturated fatty acids in a cohort of patients with cardiovascular disease. Lipids 43:289-299

27. Schaeffer L, Gohlke H, Muller M et al (2006) Common genetic variants of the FADS1 FADS2 gene cluster and their reconstructed haplotypes are associated with the fatty acid composition in phospholipids. Hum Mol Genet 15:1745-1756

28. Wei LJ, Lin DY, Weissfeld L (1989) Regression analysis of multivariate incomplete failure time data by modeling marginal distributions. J Am Stat Assoc 84:1065-1073

29. Barlow WE (1994) Robust variance estimation for the case-cohort design. Biometrics 50:1064-1072

30. Barlow WE, Ichikawa L, Rosner D, Izumi S (1999) Analysis of casecohort designs. J Clin Epidemiol 52:1165-1172

31. Rothman KJ (1990) No adjustments are needed for multiple comparisons. Epidemiology 1:43-46

32. Serhan C (2007) Resolution phase of inflammation: novel endogenous anti-inflammatory and proresolving lipid mediators and pathways. Annu Rev Immunol 25:101-137

33. Kaur G, Cameron-Smith D, Garg M, Sinclair AJ (2011) Docosapentaenoic acid (22:5n-3): a review of its biological effects. Prog Lipid Res 50: 28-34

34. Akiba S, Murata T, Kitatani K, Sato T (2000) Involvement of lipoxygenase pathway in docosapentaenoic acid-induced inhibition of platelet aggregation. Biol Pharm Bull 23:1293-1297

35. Norris PC, Dennis EA (2012) Omega-3 fatty acids cause dramatic changes in TLR4 and purinergic eicosanoid signaling. Proc Natl Acad Sci U S A 109:8517-8522

36. Oda E, Hatada K, Katoh K, Kodama M, Nakamura Y, Aizawa Y (2005) A case-control pilot study on n-3 polyunsaturated fatty acid as a negative risk factor for myocardial infarction. Int Heart J 46:583-591

37. Rissanen T, Voutilainen S, Nyyssonen K, Lakka TA, Salonen JT (2000) Fish oil-derived fatty acids, docosahexaenoic acid and docosapentaenoic acid, and the risk of acute coronary events: the Kuopio ischaemic heart disease risk factor study. Circulation 102: 2677-2679
38. Rhee Y, Paik MJ, Kim KR et al (2008) Plasma free fatty acid level patterns according to cardiovascular risk status in postmenopausal women. Clin Chim Acta 392:11-16

39. Virtanen JK, Nyantika AN, Kauhanen J, Voutilainen S, Tuomainen TP (2012) Serum long-chain n-3 polyunsaturated fatty acids, methylmercury and blood pressure in an older population. Hypertens Res Off J Japan Soc Hypertens 35:1000-1004

40. Virtanen SM, Niinisto S, Nevalainen J et al (2010) Serum fatty acids and risk of advanced beta-cell autoimmunity: a nested case-control study among children with HLA-conferred susceptibility to type I diabetes. Eur J Clin Nutr 64:792-799

41. Baur LA, O'Connor J, Pan DA, Wu BJ, O'Connor MJ, Storlien LH (2000) Relationships between the fatty acid composition of muscle and erythrocyte membrane phospholipid in young children and the effect of type of infant feeding. Lipids 35:77-82

42. Moilanen T (1987) Short-term biological reproducibility of serum fatty acid composition in children. Lipids 22:250-252

43. Katan MB, Deslypere JP, van Birgelen AP, Penders M, Zegwaard M (1997) Kinetics of the incorporation of dietary fatty acids into serum cholesteryl esters, erythrocyte membranes, and adipose tissue: an 18month controlled study. J Lipid Res 38:2012-2022

44. Arab L (2003) Biomarkers of fat and fatty acid intake. J Nutr 133(Suppl 3):925S-932S

45. Benistant C, Achard F, Ben SS, Lagarde M (1996) Docosapentaenoic acid $(22: 5, \mathrm{n}-3)$ : metabolism and effect on prostacyclin production in endothelial cells. Prostaglandins Leukot Essent Fatty Acids 55:287292

46. Burdge GC, Calder PC (2005) Conversion of alpha-linolenic acid to longer-chain polyunsaturated fatty acids in human adults. Reprod Nutr Dev 45:581-597

47. Hellstrand S, Sonestedt E, Ericson U et al (2012) Intake levels of dietary long-chain PUFAs modify the association between genetic variation in FADS and LDL-C. J Lipid Res 53:1183-1189

48. Dumont J, Huybrechts I, Spinneker A et al (2011) FADS1 genetic variability interacts with dietary alpha-linolenic acid intake to affect serum non-HDL-cholesterol concentrations in European adolescents. J Nutr 141:1247-1253

49. Lu Y, Feskens EJ, Dolle ME et al (2010) Dietary n-3 and n-6 polyunsaturated fatty acid intake interacts with FADS1 genetic variation to affect total and HDL-cholesterol concentrations in the Doetinchem Cohort Study. Am J Clin Nutr 92:258-265

50. Standl M, Lattka E, Stach B et al (2012) FADS1 FADS2 gene cluster, PUFA intake and blood lipids in children: results from the GINIplus and LISAplus studies. PLoS One 7:e37780 